\title{
LIOUVILLIAN AND ANALYTIC INTEGRABILITY OF THE QUADRATIC VECTOR FIELDS HAVING AN INVARIANT ELLIPSE
}

\author{
JAUME LLIBRE ${ }^{1}$ AND CLÀUDIA VALLS ${ }^{2}$
}

\begin{abstract}
We characterize the Liouvillian and analytic integrability of the quadratic polynomial vector fields in $\mathbb{R}^{2}$ having an invariant ellipse. More precisely, a quadratic system having an invariant ellipse can be written into the form $\dot{x}=$ $x^{2}+y^{2}-1+y(a x+b y+c), \dot{y}=-x(a x+b y+c)$, and the ellipse becomes $x^{2}+y^{2}=1$. We prove that

(i) this quadratic system is analytic integrable if and only if $a=0$;

(ii) if $x^{2}+y^{2}=1$ is a periodic orbit, then this quadratic system is Liouvillian integrable if and only if $x^{2}+y^{2}=1$ is not a limit cycle; and

(iii) if $x^{2}+y^{2}=1$ is not a periodic orbit, then this quadratic system is Liouvilian integrable if and only if $a=0$.
\end{abstract}

\section{Introduction AND STATEMENT OF THE MAIN RESUlts}

We study polynomial differential systems in $\mathbb{R}^{2}$ defined by

$$
\dot{x}=P(x, y), \quad \dot{y}=Q(x, y),
$$

where $P$ and $Q$ are polynomials with real coefficients such that the maximum degree of $P$ and $Q$ is at most $m$. When $m=2$ we call these differential systems simply quadratic systems. The dot denotes derivative with respect to the independent variable $t$, which is called here the time. Associated with system (1) we have the quadratic polynomial vector field $\mathcal{X}$ with

$$
\mathcal{X}=P(x, y) \frac{\partial}{\partial x}+Q(x, y) \frac{\partial}{\partial y} .
$$

We also refer to $\mathcal{X}$ as a quadratic vector field. For general properties on quadratic systems see $[1,14]$.

In spite of over a thousand papers published on quadratic systems (see for instance [11] or [14]) questions about their integrability are hard to tackle. Here we complete the characterization of the Liouvillian and analytic integrability of the quadratic systems having an invariant ellipse.

An isolated singular point of a quadratic system is called a center if it has a neighborhood such that every solution passing through one of its points other than the singularity is a non-trivial periodic solution. The set of all periodic orbits surrounding a given center is called the period annulus of that center.

2010 Mathematics Subject Classification. Primary 34C05, 34A34, 34C14.

Key words and phrases. Liouvillian integrability, quadratic planar polynomial vector fields, invariant ellipse. 
A limit cycle of a differential system (1) is a periodic orbit isolated in the set of all periodic orbits of system (1).

As usual $\mathbb{C}[x, y]$ denotes the ring of all complex polynomials in the variables $x$ and $y$. Let $f(x, y) \in \mathbb{C}[x, y]$. We say that $f=0$ is an invariant algebraic curve of the polynomial vector field $\mathcal{X}$ if it satisfies

$$
\mathcal{X} f=P \frac{\partial f}{\partial x}+Q \frac{\partial f}{\partial y}=K f
$$

the polynomial $K=K(x, y) \in \mathbb{C}[x, y]$ is called the cofactor of $f=0$. It has degree at most $m-1$. When $f=0$ is an ellipse and $f=0$ is an invariant algebraic curve, we will say simply that $f=0$ is an invariant ellipse. Moreover if $f(x, y) \notin$ $\mathbb{R}[x, y]$ then the conjugate of $f(x, y)$ provides also an invariant algebraic curve with cofactor the conjugate of $K(x, y)$. Independently of working with real polynomial vector fields $\mathcal{X}$ when we study their Liouvillian first integrability we need to consider the complex invariant algebraic curves, because sometimes these complex invariant algebraic curves force the real integrability.

An exponential factor $F$ of the polynomial vector field $\mathcal{X}$ of degree $m$ is an exponential function of the form $F=\exp (h / g) \notin \mathbb{C}$ with $g, h \in \mathbb{C}[x, y]$ satisfying

$$
\mathcal{X} F=P \frac{\partial F}{\partial x}+Q \frac{\partial F}{\partial y}=L F,
$$

for some polynomial $L$ of degree at most $m-1$, called the cofactor of the exponential factor $F$. Exponential factors of the form $\exp (h / g)$ with $g \neq 1$ appear when the multiplicity of the invariant algebraic curve $g=0$ is larger than one, and with $g=1$ appear when the multiplicity of the invariant straight line at infinity is larger than one, see for more details [6].

Let $U$ be an open and dense set in $\mathbb{R}^{2}$. We say that a non-constant $C^{1}$ function $H: U \rightarrow \mathbb{R}$ is a first integral of the polynomial vector field $\mathcal{X}$ on $U$, if $H(x(t), y(t))$ is constant for all values of $t$ for which the solution $(x(t), y(t))$ of $\mathcal{X}$ is defined on $U$. Clearly $H$ is a first integral of $\mathcal{X}$ on $U$ if and only if $\mathcal{X} H=0$ on $U$. We say that it is an analytic first integral if the function $H$ is analytic.

A non-constant complex function $R: U \rightarrow \mathbb{R}$ is an integrating factor for the polynomial vector field $\mathcal{X}$, if one of the following three equivalent conditions holds

$$
\frac{\partial(R P)}{\partial x}=-\frac{\partial(R Q)}{\partial y}, \quad \operatorname{div}(R P, R Q)=0, \quad \mathcal{X} R=-R \operatorname{div}(P, Q)
$$

on $U$. As usual the divergence of the vector field $\mathcal{X}$ is defined by

$$
\operatorname{div}(P, Q)=\frac{\partial P}{\partial x}+\frac{\partial Q}{\partial y}
$$

Knowing an integrating factor $R$ of (1) we can compute a first integral $H$ of $\mathcal{X}$ as

$$
H=-\int R(x, y) P(x, y) d y+h(x)
$$

where the function $h(x)$ is determined from the equality $\frac{\partial H}{\partial x}=R(x, y) Q(x, y)$. 
Let $f_{i}, g_{j}, h_{j} \in \mathbb{C}[x, y]$ for $i=1, \ldots, p$ and $j=1, \ldots, q$. Then the (multi-valued) function

$$
f_{1}^{\lambda_{1}} \cdots f_{p}^{\lambda_{p}} e^{\mu_{1} g_{1} / h_{1}} \cdots e^{\mu_{q} g_{q} / h_{q}}
$$

with $\lambda_{i}, \mu_{j} \in \mathbb{C}$ is called a generalized Darboux function.

We say that a polynomial differential system (1) is Liouvillian integrable if it has a first integral or an integrating factor given by a generalized Darboux function. Roughly speaking Liouvillian integrable polynomial differential systems are polynomial differential systems having first integrals given by elementary functions or by integrals of elementary functions, for more details see Singer [13].

It is well-known that doing an affine change of variables in $\mathbb{R}^{2}$ a quadratic system having an invariant ellipse can be written into the form

$$
\begin{aligned}
& \dot{x}=x^{2}+y^{2}-1+y(a x+b y+c), \\
& \dot{y}=-x(a x+b y+c),
\end{aligned}
$$

and the ellipse becomes $x^{2}+y^{2}=1$, the unit circle. For more details see [14] or [12]. The next result characterizes the quadratic systems which are analytic integrable.

Theorem 1. The quadratic system (6) is analytic integrable if and only if $a=0$.

The proof of Theorem 1 is given in section 2 .

The next result characterizes the quadratic systems having an invariant ellipse which are Liouvillian integrable.

Theorem 2. Consider the quadratic system (6) with the invariant ellipse $x^{2}+y^{2}=1$.

(a) Assume that $x^{2}+y^{2}=1$ is a periodic orbit. Then system (6) is Liouvillian integrable if and only if $x^{2}+y^{2}=1$ is not a limit cycle.

(b) Assume that $x^{2}+y^{2}=1$ is not a periodic orbit. Then system (6) is Liouvilian integrable if and only if $a=0$.

Chavarriga, Giacomini and Grau in [3] proved that if $x^{2}+y^{2}=1$ is a limit cycle then system (6) is not Liouvillian integrable. Llibre and Schlomiuk in [12] proved that if $x^{2}+y^{2}=1$ is a periodic orbit but not a limit cycle, then $x^{2}+y^{2}=1$ is contained in the period annulus of a center. It is well-known that quadratic systems having a center are Liouvillian integrable, see [12] and the references quoted therein, or see section 8.7 of [8]. In short, statement (a) of Theorem 2 is proved.

The proof of statement (b) of Theorem 2 is given in section 3 .

\section{Proof of Theorem 1}

Note that when $a=0$ system (6) has the first integral

$$
H=(c+b y)^{2}\left(x^{2}+y^{2}-1\right)^{b}
$$

which is an analytic first integral. Hence from now on we will assume that $a \neq 0$.

We introduce the change of variables

$$
X=x+i y, \quad Y=x-i y .
$$


With this change of variables system (6) becomes

$$
\begin{aligned}
& \dot{x}=x\left(y-\frac{i a}{2}(x+y)-\frac{b}{2}(x-y)-i c\right)-1, \\
& \dot{y}=y\left(x+\frac{i a}{2}(x+y)+\frac{b}{2}(x-y)+i c\right)-1,
\end{aligned}
$$

where we have denoted again $(x, y)$ instead of $(X, Y)$. The proof of Theorem 1 will be an immediate consequence of the following lemma.

Lemma 3. System (8) with $a \neq 0$ has no analytic first integrals.

Proof. Let $f=f(x, y)$ be an analytic first integral of system (8).

We do the change of variables $(x, y) \rightarrow(z, y)$ with $z=x y-1$. Then system (8) becomes

$$
\begin{aligned}
& \dot{z}=z\left(\frac{z+1}{y}+y\right), \\
& \dot{y}=y\left(\frac{z}{y}+\frac{i a}{2}\left(\frac{z+1}{y}+y\right)+\frac{b}{2}\left(\frac{z+1}{y}-y\right)+i c\right) .
\end{aligned}
$$

Let $g(z, y)=f\left(\frac{z+1}{y}, y\right)$. Note that $g$ is a analytic in the variable $z$ with coefficients being a Laurent series around zero in the variable $y$. Moreover $g$ satisfies

$$
z\left(\frac{z+1}{y}+y\right) \frac{\partial g}{\partial z}+y\left(\frac{z}{y}+\frac{i a}{2}\left(\frac{z+1}{y}+y\right)+\frac{b}{2}\left(\frac{z+1}{y}-y\right)+i c\right) \frac{\partial g}{\partial y}=0 .
$$

We restrict the equality to $z=0$, and denoting $\bar{g}(y)=g(0, y)$ we get

$$
\left(\frac{i a}{2}\left(1+y^{2}\right)+\frac{b}{2}\left(1-y^{2}\right)+i c y\right) \frac{d \bar{g}}{d y}=0
$$

that is $\bar{g}$ is a constant, that can be always taken to be equal zero. Therefore, $g=z g_{1}$ for some function $g_{1}$ (which is analytic in $z$ with coefficients Laurent series around zero in $y$ ) that, after simplifying by $z$, satisfies

$$
\begin{aligned}
& z\left(\frac{z+1}{y}+y\right) \frac{\partial g_{1}}{\partial z}+y\left(\frac{z}{y}+\frac{i a}{2}\left(\frac{z+1}{y}+y\right)+\frac{b}{2}\left(\frac{z+1}{y}-y\right)+i c\right) \frac{\partial g_{1}}{\partial y} \\
& =-\left(\frac{z+1}{y}+y\right) g_{1} .
\end{aligned}
$$

We consider two different cases.

Case 1: $g_{1}$ is not divisible by $z$. In this case if we denote by $\bar{g}_{1}$ the restriction of $g_{1}$ to $z=0$, then we have that $\bar{g}_{1} \neq 0$ and $\bar{g}_{1}$ satisfies

$$
\left(\frac{i a}{2}\left(1+y^{2}\right)+\frac{b}{2}\left(1-y^{2}\right)+i c y\right) \frac{d \bar{g}_{1}}{d y}=-\frac{1+y^{2}}{y} \bar{g}_{1} .
$$

Solving it we get $\bar{g}_{1}=C \exp \left(h_{1} / h_{2}\right)$ where $C \in \mathbb{C} \backslash\{0\}$ and 
If $c^{2} \neq a^{2}+b^{2}$, then $h_{2}=a^{2}+b^{2}$ and

$$
\begin{aligned}
h_{1}= & \frac{4 i a c \tanh ^{-1}\left(\frac{c+(a+i b) y}{\sqrt{S}}\right)}{\sqrt{S}}+2 i a \log y+b\left(2 i \tan ^{-1}\left(\frac{b\left(y^{2}-1\right)}{a y^{2}+2 c y+a}\right)\right. \\
& \left.-2 \log y+\log \left(4 c^{2} y^{2}+4 a c\left(y^{2}+1\right) y+b^{2}\left(y^{2}-1\right)^{2}+a^{2}\left(y^{2}+1\right)^{2}\right)\right) .
\end{aligned}
$$

If $c^{2}=a^{2}+b^{2}$, then $h_{2}=-(b+i a)$ and

$$
\begin{aligned}
h_{1}= & \frac{4 a\left(-a+i b+\sqrt{a^{2}+b^{2}} y\right)}{\left(y^{2}-1\right) a^{2}+2 i b y^{2} a-b^{2}\left(y^{2}+1\right)}+\frac{4 i b \sqrt{a^{2}+b^{2}} \tan ^{-1}\left(\frac{\sqrt{a+i b y}}{\sqrt{i b-a}}\right)}{\sqrt{i b-a}(a+i b)^{3 / 2}} \\
& -\frac{2 b \tan ^{-1}\left(\frac{a\left(y^{2}-1\right)}{b y^{2}+b}\right)}{a+i b}+2 \log y-\frac{i b \log \left(a^{2}\left(y^{2}-1\right)^{2}+b^{2}\left(y^{2}+1\right)^{2}\right)}{a+i b} .
\end{aligned}
$$

Note that $\bar{g}_{1}=\tilde{g}_{1}(y) y^{-2 /(b+i a)}$, where $\tilde{g}_{1}$ is analytic. Since $\bar{g}_{1}$ must be a Laurent series around zero in the variable $y$ and $a \neq 0$ we get that $\bar{g}_{1}=0$ which is not possible.

Case 2: $g_{1}$ is divisible by $z$. Let $n$ be the degree of $g_{1}$ in the variable $z$. In this case we write $g_{1}=z^{j} g_{j}$ for some $1 \leq j \leq n$ and such that $g_{j}$ is not divisible by $z$, i.e., if we denote by $\bar{g}_{j}$ the restriction of $g_{j}$ to $z=0$, then we have $\bar{g}_{j} \neq 0$. Moreover, $g_{j}$ satisfies

$$
\begin{aligned}
& z\left(\frac{z+1}{y}+y\right) \frac{\partial g_{j}}{\partial z}+y\left(\frac{z}{y}+\frac{i a}{2}\left(\frac{z+1}{y}+y\right)+\frac{b}{2}\left(\frac{z+1}{y}-y\right)+i c\right) \frac{\partial g_{j}}{\partial y} \\
& =-j\left(\frac{z+1}{y}+y\right) g_{j} .
\end{aligned}
$$

If we denote by $\bar{g}_{j}$ the restriction of $g_{j}$ to $z=0$, then $\bar{g}_{j} \neq 0$ and it satisfies

$$
\left(\frac{i a}{2}\left(1+y^{2}\right)+\frac{b}{2}\left(1-y^{2}\right)+i c y\right) \frac{d \bar{g}_{j}}{d y}=-j \frac{1+y^{2}}{y} \bar{g}_{j} .
$$

Solving it we get $\bar{g}_{j}=C \exp \left(h_{1} / h_{2}\right)$ where $C \in \mathbb{C} \backslash\{0\}$.

If $c^{2} \neq a^{2}+b^{2}$, then $h_{2}=a^{2}+b^{2}$ and

$$
\begin{aligned}
h_{1} & =j\left(\frac{4 i a c \tanh ^{-1}\left(\frac{c+(a+i b) y}{\sqrt{S}}\right)}{\sqrt{S}}+2 i a \log y+b\left(2 i \tan ^{-1}\left(\frac{b\left(y^{2}-1\right)}{a y^{2}+2 c y+a}\right)\right.\right. \\
& \left.\left.-2 \log y+\log \left(4 c^{2} y^{2}+4 a c\left(y^{2}+1\right) y+b^{2}\left(y^{2}-1\right)^{2}+a^{2}\left(y^{2}+1\right)^{2}\right)\right)\right) .
\end{aligned}
$$


If $c^{2}=a^{2}+b^{2}$, then $h_{2}=-(b+i a)$ and

$$
\begin{aligned}
h_{1}= & j\left(\frac{4 a\left(-a+i b+\sqrt{a^{2}+b^{2}} y\right)}{\left(y^{2}-1\right) a^{2}+2 i b y^{2} a-b^{2}\left(y^{2}+1\right)}+\frac{4 i b \sqrt{a^{2}+b^{2}} \tan ^{-1}\left(\frac{\sqrt{a+i b} y}{\sqrt{i b-a}}\right)}{\sqrt{i b-a}(a+i b)^{3 / 2}}\right. \\
& \left.-\frac{2 b \tan ^{-1}\left(\frac{a\left(y^{2}-1\right)}{b y^{2}+b}\right)}{a+i b}+2 \log y-\frac{i b \log \left(a^{2}\left(y^{2}-1\right)^{2}+b^{2}\left(y^{2}+1\right)^{2}\right)}{a+i b}\right) .
\end{aligned}
$$

Note that $\bar{g}_{j}=\tilde{g}_{j}(y) y^{-2 j /(b+i a)}$, where $\tilde{g}_{j}$ is analytic. Since $\bar{g}_{j}$ must be a Laurent series around zero in the variable $y$ and $a \neq 0$ we get that $\bar{g}_{j}=0$ which is not possible This completes the proof of the lemma.

\section{Proof of statement (b) of Theorem 2}

Note that when $a=0$, system (6) has the first integral

$$
H=(c+b y)^{2}\left(x^{2}+y^{2}-1\right)^{b}
$$

which is a Liouvillian first integral because $c+b y=0$ and $x^{2}+y^{2}-1=0$ are invariant algebraic curves of system (6) when $a=0$, and consequently the function $H$ is a generalized Darboux function. Hence from now on we will assume that $a \neq 0$.

If the invariant ellipse $x^{2}+y^{2}=1$ is not a periodic orbit of system (6), then there is a singular point on $x^{2}+y^{2}=1$. Now we study the singular points of system (6). Denote by $S=a^{2}+b^{2}-c^{2}$. We have four cases.

Case 1: $b \neq-1$ and $S>0$. In this case system (6) has the four singular points

$$
p_{1,2}=\left(0, \frac{-c \pm \sqrt{c^{2}+4(b+1)}}{2(b+1)}\right), \quad p_{3,4}=\left(\frac{-a c \mp b \sqrt{S}}{a^{2}+b^{2}}, \frac{-b c \pm a \sqrt{S}}{a^{2}+b^{2}}\right) .
$$

Note that $p_{3}$ and $p_{4}$ are in $x^{2}+y^{2}=1$.

Case $2: b \neq-1$ and $S=0$. In this case system (6) has the three singular points

$$
p_{1,2}=\left(0, \frac{-c \pm \sqrt{c^{2}+4(b+1)}}{2(b+1)}\right), \quad p_{3}=\left(\frac{-a c}{a^{2}+b^{2}}, \frac{-b c}{a^{2}+b^{2}}\right) .
$$

Note that $p_{3}$ is in $x^{2}+y^{2}=1$.

Case 3: $b=-1$ and $S>0$. In this case system (6) has the three singular points

$$
q_{1}=\left(0, \frac{1}{c}\right), \quad q_{2,3}=\left(\frac{-a c \mp b \sqrt{S}}{a^{2}+1}, \frac{c \pm a \sqrt{S}}{a^{2}+1}\right) .
$$

Note that $q_{2}$ and $q_{3}$ are in $x^{2}+y^{2}=1$.

Case 4: $b=-1$ and $S=0$. In this case system (6) has the two singular points

$$
q_{1}=\left(0, \frac{1}{c}\right), \quad q_{2}=\left(\frac{-a c}{a^{2}+1}, \frac{c}{a^{2}+1}\right) .
$$

Note that $q_{2}$ is in $x^{2}+y^{2}=1$. 
In short, for proving statement (b) of Theorem 2 it is sufficient to work with $a \neq 0$ and $a^{2}+b^{2} \geq c^{2}$, otherwise there are no singular points on $x^{2}+y^{2}=1$, and consequently $x^{2}+y^{2}=1$ is a periodic orbit. The statement (b) of Theorem 2 follows directly from the following result.

Proposition 4. System (6) with $a \neq 0$ and $a^{2}+b^{2} \geq c^{2}$ has no Liouvillian first integrals.

We introduce the change of variables in (7) and we work with system (8). Note that the invariant ellipse $f=x^{2}+y^{2}-1=0$ of system (6) with non-zero cofactor $2 x$, in system (6) becomes $f=x y-1=0$ with the non-zero cofactor $K=x+y$.

We first compute the algebraic invariant curves of system (8). Note that by Lemma 3 , system (8) has no polynomial first integrals. Now we study the invariant algebraic curves with non-zero cofactor. We state an auxiliary result.

Proposition $5([5])$. Assume $f \in \mathbb{C}[x, y]$ and let $f=f_{1}^{n_{1}} \cdots f_{r}^{n_{r}}$ be its factorization in irreducible factors over $\mathbb{C}[x, y]$. Then, for a polynomial system (1), $f=0$ is an invariant algebraic curve with cofactor $K_{f}$ if and only if $f_{i}=0$ is an invariant algebraic curve for each $i=1, \ldots, r$ with cofactor $K_{f_{i}}$. Moreover $K_{f}=n_{1} K_{f_{1}}+\cdots+$ $n_{r} K_{f_{r}}$.

Proposition 5 states that to characterize the invariant algebraic curves $f=0$ it is enough to characterize the irreducible ones, that is the ones such that $f$ is irreducible. Now we study the invariant algebraic curves with non-zero cofactor.

Lemma 6. Let $f=0$ be an irreducible invariant algebraic curve polynomial of system

$$
x^{\prime}=x\left(y-\frac{i a}{2}(x+y)-\frac{b}{2}(x-y)\right), \quad y^{\prime}=y\left(x+\frac{i a}{2}(x+y)+\frac{b}{2}(x-y)\right)
$$

with cofactor $K=\beta_{0}+\beta_{1} x+\beta_{2} y$. Then

$$
\beta_{0}=0, \quad \beta_{1}=-\frac{i l(a-i b)}{2} \text { and } \quad \beta_{2}=\frac{i l(a+i b)}{2},
$$

for some non-negative integer $l$.

Proof. We note that $x=0, y=0$ and $(a-i(b+1)) x+(a+i(b+1)) y=0$ are invariant algebraic curves of system (14) with non-zero cofactors.

Under the assumptions of Lemma 6 , if we denote by $\bar{f}=\bar{f}(y)$ the restriction of $f$ to $x=0$ then $\bar{f} \neq 0$ (otherwise would be reducible) and it satisfies

$$
\left(\frac{i a}{2}-\frac{b}{2}\right) y^{2} \frac{d \bar{f}}{d y}=\left(\beta_{0}+\beta_{2} y\right) \bar{f} \text {. }
$$

Then

$$
\bar{f}=K e^{\frac{2 i \beta_{0}}{(a+i b) y}} y^{\frac{-2 i \beta_{2}}{a+i b}}, \quad K \in \mathbb{C} \backslash\{0\} .
$$

Then since $\bar{f}$ must be a polynomial we must have $\beta_{0}=0$ and $\beta_{2}=\frac{i l(a+i b)}{2}$ for some non-negative integer $l$. 
If we denote by $\tilde{f}=\tilde{f}(x)$ the restriction of $f$ to $y=0$ then $\tilde{f} \neq 0$ (otherwise would be reducible) and we have, after simplifying by $x$,

$$
-\left(\frac{i a}{2}+\frac{b}{2}\right) x \frac{d \tilde{f}}{d x}=\beta_{1} \tilde{f}
$$

Then

$$
\tilde{f}=K y^{\frac{2 i \beta_{1}}{a-i b}}, \quad K \in \mathbb{C} \backslash\{0\} .
$$

Then since $\tilde{f}$ must be a polynomial we must have $\beta_{1}=-\frac{i j(a-i b)}{2}$ for some nonnegative integer $j$.

Now we denote by $\hat{f}=\hat{f}(y)$ the restriction of $f$ to $x=-\frac{a+i(b+1)}{a-i(b+1)} y$. Then we have that $\hat{f} \neq 0$ (otherwise would be reducible) and it satisfies, after simplifying by $y$,

Then

$$
\frac{(1+b) y}{1+b+i a} y \frac{d \hat{f}}{d y}=\frac{i\left(a(j-l)+a^{2}(j+l)+b(1+b)(j+l)\right)}{2(a-i(1+b))} \hat{f} .
$$

$$
\hat{f}=K y^{\frac{-i a(j-l)+a^{2}(j+l)+b(1+b)(j+l)}{2(1+b)}}, \quad K \in \mathbb{C} \backslash\{0\} .
$$

Since $\hat{f}$ must be a polynomial and $a \in \mathbb{R}, j, l \in \mathbb{N}$ we get that $j=l$. This completes the proof of the lemma.

Lemma 7. The unique irreducible invariant algebraic curves of system (8) with nonzero cofactor is $f=x y-1=0$.

Proof. Since the degree of system (8) is two, the degree of any cofactor is at most one, that is it can be written into the form

$$
K=\alpha_{0}+\alpha_{1} x+\alpha_{2} y, \quad\left(\alpha_{0}, \alpha_{1}, \alpha_{2}\right) \in \mathbb{C}^{3} \backslash\{(0,0,0)\} .
$$

It follows by direct computations that system (8) has no invariant algebraic curves of degree one and that the only irreducible invariant algebraic curves of degree two is $x y-1=0$. Now we shall prove that this is the only irreducible invariant algebraic curve. We proceed by contradiction.

Assume that $f=f(x, y)=0$ is an irreducible invariant algebraic curve of system (8) with non-zero cofactor $K$ as above. Then it satisfies

$$
\begin{aligned}
& \left(x\left(y-\frac{i a}{2}(x+y)-\frac{b}{2}(x-y)-i c\right)-1\right) \frac{\partial f}{\partial x} \\
& +\left(y\left(x+\frac{i a}{2}(x+y)+\frac{b}{2}(x-y)+i c\right)-1\right) \frac{\partial f}{\partial y}=\left(\alpha_{0}+\alpha_{1} x+\alpha_{2} y\right) f .
\end{aligned}
$$

We do again the change of variables $(x, y) \rightarrow(z, y)$ with $z=x y-1$. Then system (8) becomes system (9). Denoting $g(z, y)=f\left(\frac{z+1}{y}, y\right)$ equation (15) becomes $z\left(\frac{z+1}{y}+y\right) \frac{\partial g}{\partial z}+y\left(\frac{z}{y}+\frac{i a}{2}\left(\frac{z+1}{y}+y\right)+\frac{b}{2}\left(\frac{z+1}{y}-y\right)+i c\right) \frac{\partial g}{\partial y}=\left(\alpha_{0}+\alpha_{1} \frac{z+1}{y}+\alpha_{2} y\right) g$. 
Now we denote by $\bar{g}$ the restriction of $g$ to $z=0$, i.e. $\bar{g}(y)=g(0, y)$. We have that $\bar{g} \neq 0$ (since otherwise would be reducible) and it satisfies

$$
\left(\frac{i a}{2}\left(1+y^{2}\right)+\frac{b}{2}\left(1-y^{2}\right)+i c y\right) \frac{d \bar{g}}{d y}=\frac{\alpha_{0} y+\alpha_{1}+\alpha_{2} y^{2}}{y} \bar{g} .
$$

We consider four different cases.

Case 1: $c^{2} \neq a^{2}+b^{2}$ and $b \neq 0$. Solving it we get

$$
\begin{aligned}
\bar{g}= & C y^{\frac{2 \alpha_{1}}{i a+b}}\left(4 c^{2} y^{2}+4 a c\left(y^{2}+1\right) y+b^{2}\left(y^{2}-1\right)^{2}+a^{2}\left(y^{2}+1\right)^{2}\right)^{\frac{i a\left(\alpha_{1}-\alpha_{2}\right)-\left(\alpha_{1}+\alpha_{2}\right) b}{2\left(a^{2}+b^{2}\right)}} \\
\times & \exp \left(\frac{a\left(\alpha_{1}-\alpha_{2}\right)+i\left(\alpha_{1}+\alpha_{2}\right) b}{a^{2}+b^{2}} \tan ^{-1}\left(\frac{b-b y^{2}}{a+2 c y+a y^{2}}\right)\right. \\
& \left.+\frac{2 i\left(a^{2} \alpha_{0}-a\left(\alpha_{1}+\alpha_{2}\right) c+b\left(\alpha_{0} b-i\left(\alpha_{1}-\alpha_{2}\right) c\right)\right.}{\sqrt{-a^{2}-b^{2}+c^{2}}\left(a^{2}+b^{2}\right)} \tanh ^{-1}\left(\frac{c+(a+i b) y)}{\sqrt{-a^{2}-b^{2}+c^{2}}}\right)\right),
\end{aligned}
$$

where $C$ is a non-zero constant. It is clear that $\bar{f}$ must be a rational function in the variable $y$. Clearly we must have

$$
a\left(\alpha_{1}-\alpha_{2}\right)+i\left(\alpha_{1}+\alpha_{2}\right) b=0 \quad \text { and } \quad a^{2} \alpha_{0}-a\left(\alpha_{1}+\alpha_{2}\right) c+b\left(\alpha_{0} b-i\left(\alpha_{1}-\alpha_{2}\right) c=0,\right.
$$

that is

$$
\alpha_{1}=\frac{a-i b}{a+i b} \alpha_{2} \quad \text { and } \quad \alpha_{0}=\frac{2 c}{a+i b} \alpha_{2}
$$

Then $\bar{g}=C y^{\frac{-2 i(a-i b) \alpha_{2}}{a^{2}+b^{2}}}$. Again since $\bar{g}$ is a rational function in the variable $y$ we have

$$
\alpha_{2}=-\frac{a+i b}{2 i} j
$$

for some non-negative integer $j$. In summary

$$
\alpha_{0}=i c j, \quad \alpha_{1}=\frac{i j}{2}(a-i b) \quad \text { and } \quad \alpha_{2}=\frac{i j}{2}(a+i b) .
$$

Now we write $f$ in sum of its homogeneous parts as $f=\sum_{i=1}^{n} f_{i}$ where each $f_{i}=$ $f_{i}(x, y)$ is a homogeneous polynomial of degree $i$. Without loss of generality we can assume that $n>0$ and $f_{n} \neq 0$. Moreover $f_{n}$ satisfies the equation

$$
\begin{aligned}
& x\left(y-\frac{i a}{2}(x+y)-\frac{b}{2}(x-y)\right) \frac{\partial f_{n}}{\partial x}+y\left(x+\frac{i a}{2}(x+y)+\frac{b}{2}(x-y)\right) \frac{\partial f_{n}}{\partial y} \\
& =\frac{i j}{2}((a-i b) x+(a+i b) y) f_{n}
\end{aligned}
$$

Since $f_{n} \neq 0$ we get that $f_{n}$ must be an invariant algebraic curve of system (14) with cofactor $K=-\frac{i j}{2}((a-i b) x+(a+i b) y)$, i.e., $\beta_{0}=0, \beta_{1}=i j(a-i b) / 2$ and $\beta_{2}=$ $i j(a+i b) / 2$. It follows from Lemma 6 that $j=0$ which yields that $\alpha_{1}=\alpha_{2}=\alpha_{3}=0$, in contradiction with the fact that the cofactor is nonzero. Hence this case is not possible. 
Case 2: $c^{2} \neq a^{2}+b^{2}$ and $b=0$. Solving it we get

$$
\begin{aligned}
\bar{g} & =C y^{\frac{2 \alpha_{1}}{i a}}\left(4 c^{2} y^{2}+4 a c\left(y^{2}+1\right) y+a^{2}\left(y^{2}+1\right)^{2}\right)^{\frac{i\left(\alpha_{1}-\alpha_{2}\right)}{2 a}} \\
& \times \exp \left(\frac{2 i\left(a \alpha_{0}-\left(\alpha_{1}+\alpha_{2}\right) c\right)}{\sqrt{-a^{2}+c^{2}} a} \tanh ^{-1}\left(\frac{c+a y}{\sqrt{-a^{2}+c^{2}}}\right)\right),
\end{aligned}
$$

where $C$ is a non-zero constant. It is clear that $\bar{f}$ must be a rational function in the variable $y$. Clearly we must have

$$
\alpha_{0}=\frac{\left(\alpha_{1}+\alpha_{2}\right) c}{a}, \quad \alpha_{1}=\frac{i a j_{1}}{2} \quad \text { and } \quad \alpha_{2}=i a\left(\frac{j_{1}}{2}+2 j_{2}\right)
$$

for some non-negative integers $j_{1}$ and $j_{2}$.

Now we write $f$ in sum of its homogeneous parts as $f=\sum_{i=1}^{n} f_{i}$ where each $f_{i}=$ $f_{i}(x, y)$ is a homogeneous polynomial of degree $i$. Again, without loss of generality we can assume that $n>0$ and $f_{n} \neq 0$. Moreover, proceeding as in Case $1, f_{n}=0$ must be an irreducible invariant algebraic curve of system (14) with $b=0$ and with cofactor $K=\frac{i a}{2}\left(j_{1} x+\left(j_{1}+4 j_{2}\right) y\right)$, i.e., $\beta_{0}=0, \beta_{1}=i j_{1} a / 2$ and $\beta_{2}=i a\left(j_{1}+4 j_{2}\right) / 2$. It follows from Lemma 6 that $j_{1}=j_{2}=0$ which yields $\alpha_{1}=\alpha_{2}=\alpha_{3}=0$, in contradiction with the fact that the cofactor is nonzero. Hence this case is not possible.

Case 3: $c^{2}=a^{2}+b^{2}$ and $b \neq 0$. Solving it we get

$$
\begin{aligned}
\bar{g}= & C y^{\frac{-2 i \alpha_{1}}{a+i b}}\left(a^{2}\left(y^{2}-1\right)^{2}+b^{2}\left(y^{2}+1\right)^{2}\right)^{\frac{i\left(a\left(\alpha_{1}-\alpha_{2}\right)+i\left(\alpha_{1}+\alpha_{2}\right) b\right)}{2(a+i b)}} \\
\times & \exp \left(\frac{a\left(\alpha_{1}-\alpha_{2}\right)+i\left(\alpha_{1}+\alpha_{2}\right) b}{(a+i b)^{2}} \tan ^{-1}\left(\frac{a\left(y^{2}-1\right)}{b y^{2}+b}\right)\right. \\
& -\frac{2\left(a\left(\alpha_{1}+\alpha_{2}\right)+i b\left(\alpha_{1}+\alpha_{2}\right)\right) \sqrt{a^{2}+b^{2}}}{\left(a^{2}+b^{2}\right)(a+i b)^{2}} \tanh ^{-1}\left(\frac{\sqrt{a+i b} y}{\sqrt{i b-a}}\right) \\
& -\frac{i}{\left(a^{2}+b^{2}-(a+i b)^{2} y^{2}\right)(a+i b)}\left(4 \left(\left(\alpha_{1}+\alpha_{2}+\alpha_{0} y\right) a^{2}\right.\right. \\
& +\left(\alpha_{2}\left(-2 i b-\sqrt{a^{2}+b^{2}} y\right)-\sqrt{a^{2}+b^{2}}\left(\alpha_{0}+\alpha_{1} y\right)\right) a \\
& \left.\left.\left.+b\left(b y \alpha_{0}+i \sqrt{a^{2}+b^{2}} \alpha_{0}-\alpha_{2} b+i \alpha_{2} \sqrt{a^{2}+b^{2}} y+\alpha_{1}\left(b-i \sqrt{a^{2}+b^{2}} y\right)\right)\right)\right)\right),
\end{aligned}
$$

where $C$ is a non-zero constant. It is clear that $\bar{f}$ must be a rational function in the variable $y$. Clearly we must have

$$
a\left(\alpha_{1}-\alpha_{2}\right)+i\left(\alpha_{1}+\alpha_{2}\right) b=0, \quad a\left(\alpha_{1}+\alpha_{2}\right)+i\left(\alpha_{1}+\alpha_{2}\right) b=0
$$

which yields $\alpha_{1}=\alpha_{2}=0$. Then, again since $\bar{f}$ must be a rational function in the variable $y$, and using that $\alpha_{1}=\alpha_{2}=0$ we must have

$$
\sqrt{a^{2}+b^{2}} \alpha_{0}(a-i b)-\alpha_{0}\left(b^{2}+4 a^{2}\right) y=0,
$$

which clearly yields $\alpha_{0}=0$, i.e. $\alpha_{1}=\alpha_{2}=\alpha_{3}=0$, in contradiction with the fact that the cofactor is nonzero. Hence this case is not possible. 
Case 4: $c^{2}=a^{2}+b^{2}$ and $b=0$. Solving it we get

$$
\begin{aligned}
\bar{g} & =C y^{-\frac{2 i \alpha_{1}}{a}}\left(y^{2}-1\right)^{-\frac{i\left(\alpha_{2}-\alpha_{1}\right)}{a}} \\
& \times \exp \left(-\frac{2 i\left(\alpha_{0}(|a|-a y)+\left(\alpha_{1}+\alpha_{2}\right)(y|a|-a)\right)}{a^{2}\left(y^{2}-1\right)}+\frac{2 i\left(\alpha_{1}-\alpha_{2}\right)|a|}{a^{2}} \tanh ^{-1} y\right),
\end{aligned}
$$

where $C$ is a non-zero constant. It is clear that $\bar{f}$ must be a rational function in the variable $y$. Clearly we must have

$$
\left.\alpha_{1}-\alpha_{2}=0, \quad \alpha_{0}(|a|-a y)+\left(\alpha_{1}+\alpha_{2}\right)(y|a|-a)\right), \quad \frac{-2 i \alpha_{1}}{a}=j
$$

for some non-negative integer $j$. This yields $\alpha_{2}=\alpha_{1}, \alpha_{0}=2|a| \alpha_{1} / a$ and $\alpha_{1}=i j a / 2$.

Now we write $f$ in sum of its homogeneous parts as $f=\sum_{i=1}^{n} f_{i}$ where each $f_{i}=$ $f_{i}(x, y)$ is a homogeneous polynomial of degree $i$. Again, without loss of generality we can assume that $n>0$ and $f_{n} \neq 0$. Moreover, proceeding as in Case $1, f_{n}=0$ must be an irreducible invariant algebraic curve of system (14) with $b=0$ and with cofactor $K=\frac{i j a}{2}(x+y)$, i.e., $\beta_{0}=0, \beta_{1}=i j a / 2$ and $\beta_{2}=i a j / 2$. It follows from Lemma 6 that $j=0$ which yields that $\alpha_{1}=\alpha_{2}=\alpha_{3}=0$, in contradiction with the fact that the cofactor is nonzero. Hence this case is not possible.

Now we present a characterization under suitable assumptions of the algebraic multiplicity of an invariant algebraic curve using the number of exponential factors.

We say that an invariant algebraic curve $f=0$ of degree $m$ of a vector field $\mathcal{X}$ has algebraic multiplicity $k$ if $f^{k}$ is a factor of the so-called extactic curve of $\mathcal{X}$ but $f^{k+1}$ is not a factor of the extactic curve, where the extactic curve of $\mathcal{X}$ is

$$
\operatorname{det}\left(\begin{array}{cccccccc}
1 & x & y & \cdots & x^{m} & x^{m-1} y & \cdots & y^{m} \\
\mathcal{X}(1) & \mathcal{X}(x) & \mathcal{X}(y) & \cdots & \mathcal{X}\left(x^{m}\right) & \mathcal{X}\left(x^{m-1} y\right) & \cdots & \mathcal{X}^{(}\left(y^{m}\right) \\
\vdots & \vdots & \vdots & \cdots & \vdots & \vdots & \vdots & \vdots \\
\mathcal{X}^{l-1}(1) & \mathcal{X}^{l-1}(x) & \mathcal{X}^{l-1}(y) & \cdots & \mathcal{X}^{l-1}\left(x^{m}\right) & \mathcal{X}^{l-1}\left(x^{m-1} y\right) & \cdots & \mathcal{X}^{l-1}\left(y^{m}\right)
\end{array}\right),
$$

where $l$ is the number of elements in the basis $\left\{1, x, y, \ldots, x^{m}, x^{m-1} y, \ldots, y^{m}\right\}$. For more details on the definition and properties of the extactic curve, see [6].

Proposition 8. For a given irreducible invariant algebraic curve $f=0$ of degree $m$ of $\mathcal{X}$. Then $f$ has algebraic multiplicity $k$ if and only if the vector field has $k-1$ exponential factors $\exp \left(g_{i} / f^{i}\right)$, where $g_{i}$ is a polynomial of degree at most im and $\left(g_{i}, f\right)=1$ for $i=1, \ldots, k-1$.

Lemma 9. System (8) has no exponential factors.

Proof. Taking into account the possible multiplicity of the line of infinity, the possible multiplicities of the finite invariant algebraic curves through Lemmas 3 and 7 , and the change of variables (7), if system (6) has exponential factors they must be of the form

$$
\exp (h), \quad \exp \left(h /\left(x^{2}+y^{2}-1\right)^{k}\right),
$$

where $h$ is a polynomial and $k$ is a positive integer. We denote by $\beta_{0}+\beta_{1} x+\beta_{2} y$ the cofactor of one of these exponential factors. 
First we shall see that there are no exponential factors of the form $\exp (h)$ with $h$ a polynomial. From the definition of exponential factor, the left hand side of equality (3) is zero on the singular points. Then, taking into account the study of the finite singular points of system (6) done in the beginning of this section we have:

Case 1: $b \neq-1$ and $S>0$. In this case

$$
\begin{aligned}
& \beta_{0}+\beta_{2}\left(\frac{-c+\sqrt{c^{2}+4(b+1)}}{2(b+1)}\right)=0, \\
& \beta_{0}+\beta_{2}\left(\frac{-c-\sqrt{c^{2}+4(b+1)}}{2(b+1)}\right)=0 \\
& \beta_{0}+\beta_{1}\left(\frac{-a c-b \sqrt{S}}{a^{2}+b^{2}}\right)+\beta_{2}\left(\frac{-b c+a \sqrt{S}}{a^{2}+b^{2}}\right)=0, \\
& \beta_{0}+\beta_{1}\left(\frac{-a c+b \sqrt{S}}{a^{2}+b^{2}}\right)+\beta_{2}\left(\frac{-b c-a \sqrt{S}}{a^{2}+b^{2}}\right)=0,
\end{aligned}
$$

where $S=a^{2}+b^{2}-c^{2}$. Solving this system we get that $\beta_{0}=\beta_{1}=\beta_{2}=0$ and thus $h$ must be a polynomial first integral, in contradiction with Lemma 3 .

Case $2: b \neq-1$ and $S=0$. In this case

$$
\begin{aligned}
& \beta_{0}+\beta_{2}\left(\frac{-c+\sqrt{c^{2}+4(b+1)}}{2(b+1)}\right)=0, \\
& \beta_{0}+\beta_{2}\left(\frac{-c-\sqrt{c^{2}+4(b+1)}}{2(b+1)}\right)=0, \\
& \beta_{0}+\beta_{1}\left(\frac{-a c}{a^{2}+b^{2}}\right)+\beta_{2}\left(\frac{-b c}{a^{2}+b^{2}}\right)=0 .
\end{aligned}
$$

Solving this system we get that $\beta_{0}=\beta_{1}=\beta_{2}=0$ and thus $h$ must be a polynomial first integral, in contradiction with Lemma 3.

Case $3: b=-1$ and $S>0$. In this case we have

$$
\begin{aligned}
& \beta_{0}+\frac{\beta_{2}}{c}=0, \\
& \beta_{0}+\beta_{1}\left(\frac{-a c+\sqrt{S}}{a^{2}+1}\right)+\beta_{2}\left(\frac{c+a \sqrt{S}}{a^{2}+1}\right)=0, \\
& \beta_{0}+\beta_{1}\left(\frac{-a c-\sqrt{S}}{a^{2}+1}\right)+\beta_{2}\left(\frac{c-a \sqrt{S}}{a^{2}+1}\right)=0,
\end{aligned}
$$

where $S=a^{2}+1-c^{2}$. Solving this system we get that $\beta_{0}=\beta_{1}=\beta_{2}=0$ and thus $h$ must be a polynomial first integral, in contradiction with Lemma 3 .

Case $4: b=-1$ and $S=0$, i.e. $b=-1$ and $c^{2}=1+a^{2}$. In this case

$$
\beta_{0}+\frac{\beta_{2}}{c}=0, \quad \beta_{0}-\frac{a}{c} \beta_{1}+\frac{\beta_{2}}{c}=0 .
$$


Solving this system we get that

$$
\beta_{0}=-\frac{\beta_{2}}{c}, \quad \beta_{1}=0
$$

Working with system (9) of the proof of Lemma 3 and denoting by $\hat{h}=\hat{h}(z, y)=$ $h(x, y)$ we obtain

$$
\text { (18) } z\left(\frac{z+1}{y}+y\right) \frac{\partial \hat{h}}{\partial z}+y\left(\frac{z}{y}+\frac{i a}{2}\left(\frac{z+1}{y}+y\right)-\frac{1}{2}\left(\frac{z+1}{y}-y\right)+i c\right) \frac{\partial \hat{h}}{\partial y}=\beta_{2}\left(-\frac{1}{c}+y\right) \text {. }
$$

Note that $\hat{h}$ is a polynomial in the variable $z$ with coefficients being rational functions in the variable $y$. Now restricting equality (18) to $z=0$ and denoting by $\bar{h}=\bar{h}(y)=$ $\hat{h}(0, y)$ we get

$$
\left(\frac{i a}{2}\left(1+y^{2}\right)-\frac{1}{2}\left(1-y^{2}\right)+i c y\right) \frac{d \bar{h}}{d y}=\beta_{2}\left(-\frac{1}{c}+y\right) .
$$

Solving it we obtain that $\bar{h}(y)$ is equal to

$$
\begin{aligned}
& \frac{2 i\left(c^{2}+a-i\right) \beta_{2} \tan ^{-1}\left(\frac{c+(a-i) y}{\sqrt{a^{2}-c^{2}+1}}\right)}{(a-i) c \sqrt{a^{2}-c^{2}+1}}-\frac{\beta_{2}}{2(a-i)}\left(2 \tan ^{-1}\left(\frac{y^{2}-1}{a y^{2}+2 c y+a}\right)\right. \\
& \left.+i \log \left(y^{4}+\left(4 c^{2}-2\right) y^{2}+4 a c\left(y^{2}+1\right) y+a^{2}\left(y^{2}+1\right)^{2}+1\right)\right) .
\end{aligned}
$$

Since $\bar{h}(y)$ must be a rational function in the variable $y, \beta_{2}=0$. So, $\beta_{0}=\beta_{1}=\beta_{2}=0$ and thus $h$ must be a polynomial first integral, in contradiction with Lemma 3 . Hence, there are no exponential factors of the form $\exp (h)$.

Now we shall see that there are no exponential factors of the form $\exp \left(h /\left(x^{2}+y^{2}-\right.\right.$ $\left.1)^{k}\right)$. In view of Proposition 8 it is enough to show it for $k=1$ and $h$ a polynomial of degree one. Thus, we take $h=\gamma_{0}+\gamma_{1} x+\gamma_{2} y$. Substituting in (3) we get

$$
\begin{aligned}
& \left(\left(x^{2}+y^{2}-1\right)+y(a x+b y+c)\right) \gamma_{1}-x(a x+b y+c) \gamma_{2}-2 x\left(\gamma_{0}+\gamma_{1} x+\gamma_{2} y\right) \\
& =\left(\beta_{0}+\beta_{1} x+\beta_{2} y\right)\left(x^{2}+y^{2}-1\right) .
\end{aligned}
$$

Solving it we get $\beta_{0}=\beta_{1}=\beta_{2}=\gamma_{0}=\gamma_{1}=\gamma_{2}=0$. Thus, $h=0$, which is not possible. This concludes the proof of the lemma.

Proof of Proposition 4. If system (6) has a Liouvillian first integral, then it has an integrating factor of the form (5) where $f_{i}=0$ are the algebraic curves and $F_{j}$ are the exponential factors of system (6). In view of Lemmas 3 and 7 we have that the unique invariant algebraic curve of system (6) is $x^{2}+y^{2}-1=0$ (a Darboux polynomial with cofactor $2 x$ ). Additionally, it follows from Lemma 9 that system (6) has no exponential factors. In short, the unique possible integrating factor must be of the form $\left(x^{2}+y^{2}-1\right)^{\lambda}$. From (4) we have

$$
2 \lambda x=-2 x+a y-b x, \quad \lambda \in \mathbb{C},
$$

which is a contradiction because $a \neq 0$. Then, system (6) has no integrating factors of the form (5). 
If system (6) has a first integral given by a function (5), from the above arguments this first integral must be $\left(x^{2}+y^{2}-1\right)^{\lambda}$. Then $\mathcal{X}\left(x^{2}+y^{2}-1\right)^{\lambda}=0$ where $\mathcal{X}$ is the vector field associated to system (6). So, we have $2 \lambda x\left(x^{2}+y^{2}-1\right)^{\lambda}=0$, i.e. $\lambda=0$ in contradiction with the fact that $\left(x^{2}+y^{2}-1\right)^{0}=1$ is a first integral. Consequently system (6) cannot have a Liouvillian first integral.

\section{ACKNowledgements}

The first author was partially supported by the MICINN/FEDER grant MTM200803437, AGAUR grant 2009SGR-410 and ICREA Academia. The second author is supported by AGAUR grant PIV-DGR-2010 and by FCT through CAMGDS, Lisbon.

\section{REFERENCES}

[1] W.A. Coppel, A survey of quadratic systems, J. Differential Equations 2 (1996), 293-304.

[2] J. Chavarriga, J. Llibre And J. Sotomayor, Algebraic solutions for polynomial vector fields with emphasis in the quadratic case, Expositiones Math. 15 (1997), 161-173.

[3] J. Chavarriga, H. Giacomini and M. Grau, Quadratic systems with an algebraic limit cycle of degree two or four don't have a Liouvillian first integral, EQUADIFF 2003, WorldSci. Publ. NJ, 2005, pp. 325-327.

[4] C. Christopher, Invariant algebraic curves and conditions for a center, Proc. Roy. Soc. Edinburgh 124A (1994), 1209-1229.

[5] C. Christopher And J. LliBRe, Integrability via invariant algebraic curves for planar polynomial differential systems, Annals of Differential Equations 16 (2000), 5-19.

[6] C. Christopher, J. Llibre And J.V. Pereira, Multiplicity of invariant algebraic curves and Darboux integrability, Pacific J. Math. 229 (2007), 63-117.

[7] G. Darboux, Mémoire sur les équations différentielles algébriques du premier ordre et du premier degrée (Mélanges), Bull. Sci. Math. 2éme série 2 (1878), 60-96; 123-144; 151-200.

[8] F. Dumortier, J. Llibre And J.C. ArtÉs, Qualitative theory of planar differential systems, UniversiText, Springer-Verlag, New York, 2006.

[9] J. Llibre And D. Schlomiuk, On the limit cycles bifurcating from an ellipse of a quadratic center, preprint.

[10] J. LLIBRE, Integrability of polynomial differential systems, in Handbook of Differential Equations, Ordinary Differential Equations, Eds. A. Cañada, P. Drabeck and A. Fonda, Elsevier, 2004, pp. 437-533.

[11] J. W. REYN, A bibliography of the qualitative theory of quadratic systems of differential equations in the plane, Delf University of Technology, http://ta.twi.tudelft.nl/DV/Staff/ J.W.Reyn.html, 1997.

[12] D. Schlomiuk, Algebraic particular integrals, integrability and the problem of the center, Trans. Amer. Math. Soc. 338 (1993), 799-841.

[13] M.F. Singer, Liouvillian first integrals of differential equations, Trans. Amer. Math. Soc. 333 (1992), 673-688.

[14] Te Yanquian et al., Theory of Limit Cycles, Translations of Math. Monographs, Vol. 66, Amer. Math. Soc. Providence, 1986.

${ }^{1}$ Departament de Matemàtiques, Universitat Autònoma de Barcelona, 08193 Bellaterra, Barcelona, Catalonia, Spain

E-mail address: jllibre@mat.uab.cat 
${ }^{2}$ Departamento de Matemática, Instituto Superior Técnico, Universidade Técnica De Lisboa, Av. Rovisco Pais 1049-001, Lisboa, Portugal

E-mail address: cvalls@math.ist.utl.pt 